\title{
DÉTECTION AUTOMATIQUE DE ZONES EN MOUVEMENT DANS DES SÉRIES D'IMAGES NON RECALÉES : APPLICATION À LA SURVEILLANCE DES MOUVEMENTS GRAVITAIRES
}

\author{
Guilhem Marsy $^{1,2,3}$, Flavien Vernier ${ }^{1}$, Xavier Bodin $^{2}$, William Castaings $^{3}$, Emmanuel Trouvé $^{1}$ \\ 1: Univ. Savoie Mont Blanc, LISTIC, 74000 Annecy, France \\ 2: CNRS, EDYTEM, 73000 Chambéry, France, France \\ 3: TENEVIA 38240 Meylan, France
}

\begin{abstract}
Résumé
Dans le domaine des géosciences, l'utilisation d'appareils photographiques automatiques fixes pour la surveillance des mouvements gravitaires est de plus en plus courante. Les séries d'images ainsi acquises permettent de suivre dans le temps l'évolution du mouvement étudié. Bien que les appareils soient fixes, on observe que les conditions du milieu extérieur peuvent entraîner un déplacement/décalage des images qui peut dépasser le déplacement dû au phénomène étudié. Nous proposons ici une méthode automatique qui analyse le déplacement apparent sur toute l'image afin de segmenter la scène en séparant les zones du terrain en mouvement des zones fixes. Nous illustrons la méthode sur une série d'images, acquises au cours de l'été 2016, du glacier rocheux du Laurichard (Hautes Alpes, France), dont les mouvements sont par ailleurs bien connus et étudiés.
\end{abstract}

Mots clés : Recalage d'images, Série temporelle d'images, Mouvements gravitaires, Segmentation de déplacements

\begin{abstract}
Within Geosciences, it is now common to use fixed time-lapse photographic cameras for studying mass movement. The series of images these equipment produce allow to monitor in time the evolution of the surface displacements. Though the device is supposed to be immobile, it is often observed that the conditions of the environment can cause a random shift of the images that may overpass the displacement amplitude of the studied phenomena. We propose here an automated method to analyze the apparent displacement over the whole image in order to segment the scene and separate between moving terrain and fixed terrain. We illustrate the method with an image series that covers the summer 2016 of the Laurichard rock glacier (France), whose dynamics and movement are well known.
\end{abstract}

Keywords : Image registration, Time-lapse, Slope movement, Displacement segmentation

\section{Introduction}

On trouve dans la littérature un grand nombre d'utilisations d'une ou plusieurs caméras fixes pour suivre des mouvements gravitaires tels que l'avancement de glaciers (Hadhri et al., 2017), glaciers rocheux (Neyer, 2016) et les glissements de terrain (Roncella et al., 2014). Ce type de dispositif permet à la fois une bonne résolution spatiale (taille du pixel au sol) et une bonne résolution temporelle (pas entre deux acquisitions d'images), et ce à moindre coût et sans installation de matériel sur le terrain en mouvement. Bien que les caméras soient le plus souvent fixées solidement à un point fixe, comme le montre la figure 1 , on observe que les variations des conditions du milieu extérieur (vent, humidité, température, rayonnement solaire...) peuvent modifier les paramètres de prise de vue, entraînant un décalage des images les unes par rapport aux autres. Selon la configuration optique du système et la distance à l'objet d'étude, ces mouvements liés à la caméra peuvent dépasser le déplacement dû au phénomène étudié. C'est pourquoi la plupart des chaînes de traitements visant à extraire un déplacement de ces séries d'images commencent par une étape de recalage d'images (Gance et al., 2014; Roncella et al., 2014; Pham, 2015; Neyer, 2016; Hadhri et al., 2017). Cette étape vise à supprimer le déplacement dû à la modification des paramètres extrinsèques et intrinsèques par le milieu extérieur. Pour ce faire, plusieurs stratégies basées sur la mise en correspondance de points peuvent être mises en œuvre :

- extraction et mise en correspondances de descripteurs ou points d'intérêts de type «Scale-Invariant Feature Transform » (SIFT) (Lowe, 2004) ou Harris (Harris et Stephens, 1988) sur les zones supposées fixes (Gance et al., 2014),

- corrélation de différentes zones supposées fixes (Pham, 2015),

- extraction manuelle de points de correspondances (Roncella et al., 2014).

Une fois la mise en correspondance de points réalisée, une homographie (matrice $3 \times 3$ ) est estimée afin de recaler l'image sur une image de référence. L'ensemble de ces méthodes sont basées sur une expertise visant à déterminer manuellement les zones fixes et mobiles. Or 


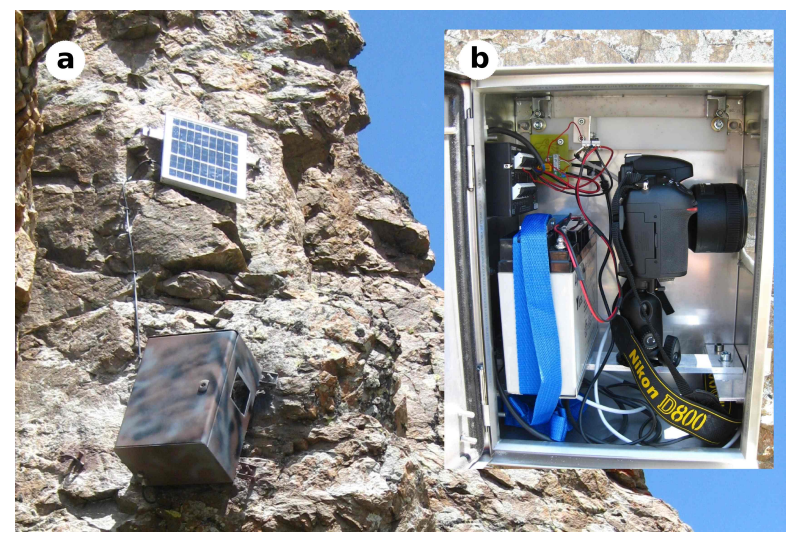

FIGURE 1: a) Présentation de l'installation de l'appareil photo sur le site du Laurichard : l'appareil photo est dans un caisson fixé à la paroi. b) À l'intérieur de la boite, l'appareil est fixé sur un pied photo.

dans certains cas, il peut être difficile d'affirmer que des zones sont fixes ou mobiles.

Les auteurs de (Dehecq et al., 2015), proposent un coefficient permettant de juger de la cohérence d'un champ de vecteurs vitesse «Velocity Vector Coherence » (VVC) et notamment de l'utiliser comme indicateur du bon recalage d'images satellites optiques. Dans cet article, nous proposons d'utiliser ce coefficient de cohérence de champ de vecteurs vitesse afin de déterminer automatiquement les zones fixes et les zones mobiles.

La suite de cet article est organisée comme suit : dans un premier temps, nous présentons la stratégie retenue afin d'extraire les champs de vecteurs vitesse dans une série d'images, puis nous détaillons l'utilisation du coefficient VVC pour la segmentation automatique du champ de vecteurs vitesse. Puis nous montrons comment cette segmentation peut être utilisée pour obtenir un champ de vecteurs vitesse représentant uniquement le déplacement étudié. Enfin, nous présentons les résultats de ces méthodes sur une série d'images du glacier rocheux du Laurichard, tout en justifiant les paramètres utilisés.

\section{Calcul du champ de vecteurs déplacement et segmentation}

\subsection{Calcul du champ de déplacement}

De façon similaire à (Neyer, 2016), nous proposons d'extraire des points caractéristiques, mais en choisissant les descripteurs «Oriented Fast and Rotated Brief 》 (ORB) (Rublee et al., 2011) sur l'intégralité des deux images entre lesquelles nous souhaitons calculer le champ de déplacement. Le choix s'est porté sur les descripteurs ORB pour leur robustesse à la rotation, et leur rapidité en termes de temps de calcul comparé aux autres méthodes de l'état de l'art comme SIFT (Lowe, 1999) ou "Speeded Up Robust Features » (SURF) (Bay et al., 2006). Ces points caractéristiques sont ensuite mis en correspondance par une méthode «brute force », puis filtrés de façon à garantir une cohérence locale. Afin de sélectionner les couples sur lesquels le traitement est effectué, nous choisissons des images suffisamment espacées dans le temps afin que le déplacement étudié soit au moins du même ordre de grandeur que le déplacement provenant du mouvement de la caméra. Nous réalisons donc le traitement sur le maximum de paires d'images disponibles satisfaisant ce critère.

\subsection{Coefficient de cohérence de vecteurs vitesse}

Le coefficient de cohérence de champ de vecteurs vitesse «Velocity Vector Coherence » (VVC) introduit dans (Dehecq et al., 2015), est défini par :

$$
V V C(i, j)=\frac{\left\|\sum_{t \in T} \vec{V}(i, j, t)\right\|}{\sum_{t \in T}\|\vec{V}(i, j, t)\|},
$$

avec $\vec{V}(i, j, t)$ le vecteur déplacement au pixel de coordonnées $\mathrm{i}, \mathrm{j}$ de l'image prise à l'instant $t$.

Ainsi sous l'hypothèse que le mouvement dû aux conditions extérieures entraînant une dilatation des lentilles de la caméra ou de la structure sur laquelle elle est fixée varie autour d'une position d'équilibre, sur un grand nombre d'images le coefficient VVC va tendre vers zéro tandis que pour des zones suivant un mouvement ne changeant pas de direction le VVC sera proche de 1. En pratique, cette hypothèse est le plus souvent vérifiée, mais il arrive que dans certains cas, le versant de montagne auquel la caméra est fixée bouge de façon significative, par exemple dans (Neyer, 2016), où l'auteur indique que le versant où se trouve une caméra surveillant un glacier rocheux se déplace de plus de $10 \mathrm{~cm}$ par an. Ainsi, dans de pareils cas, il n'est pas possible d'appliquer la méthode que nous présentons. À partir du calcul de ce coefficient sur l'ensemble de l'image à l'aide d'une série importante d'images, on peut segmenter simplement les zones fixes des zones mobiles en appliquant un seuil sur le coefficient VVC. Cependant, dans notre cas nous ne possédons pas une mesure assez dense de déplacement pour calculer le coefficient de cohérence de façon fiable pour l'ensemble des pixels de l'image. C'est pourquoi nous proposons de ne pas calculer le coefficient VVC pour chaque pixel, mais sur des fenêtres de pixels, ainsi le coefficient VVC sur une fenêtre $w_{l}$ de taille $w \times w$ donne :

$$
V V C\left(w_{l}\right)=\frac{\left\|\sum_{t \in T} \sum_{i, j \in w_{l}} \vec{V}(i, j, t)\right\|}{\sum_{t \in T} \sum_{i, j \in w_{l}}\|\vec{V}(i, j, t)\|},
$$

avec $\vec{V}(i, j, t)=\overrightarrow{0}$ si la vitesse n'est pas définie. La taille de la fenêtre $w$ doit être choisie empiriquement par rapport à la densité de vecteurs déplacements extraits par la méthode de la section 2.1 , densité qui dépend de la nature des images.

\subsection{Détermination du masque des zones mobiles}

Comme mentionné dans la section 2.2, le coefficient VVC est un indicateur des zones effectivement en mouvement : un coefficient élevé indique des zones en déplacement homogène dans le temps alors qu'un coefficient proche de zéro indique une zone fixe. II faut donc 
choisir un seuil sur le coefficient VVC au-dessus duquel la zone est considérée comme en mouvement. La détermination de ce seuil est critique pour une bonne segmentation et son influence sera présentée dans la section 3.3. Après analyse de l'histogramme du coefficient VVC calculé sur des séries d'images, il s'avère que l'histogramme présente deux modes écartés par une large vallée, le second mode correspondant aux valeurs de VVC associées à des zones en mouvements. On trouve un exemple d'histogramme du coefficient VVC dans la figure 2.5. Pour déterminer automatiquement le seuil entre ces deux modes nous avons utilisé une méthode dite de Huang, proposée dans (Huang et Wang, 1995), particulièrement adapté à la segmentation d'images dont les histogrammes présentent deux modes séparés par une large vallée. Enfin, afin d'améliorer la qualité de ce masque, des opérations morphologiques peuvent être appliquées pour réduire le bruit présent dans le masque.

\section{Application au glacier rocheux du Laurichard}

\subsection{Glacier rocheux}

Caractéristiques des milieux à permafrost, les glaciers rocheux sont très fréquents sur les versants alpins situés au-delà de $2500 \mathrm{~m}$ d'altitude. Ils sont constitués de masses de débris rocheux contenant en leur sein de la glace en quantité suffisante pour que l'ensemble soit animé d'un mouvement lent et continu (de l'ordre de quelques $\mathrm{cm}$ à quelques m/a) vers l'aval (Haeberli et al., 2006). Cette déformation confère aux glaciers rocheux une morphologie caractéristique, avec un aspect général d'une langue rayée de sillons arqués et délimités par des talus raides. La présence de débris rocheux en surface crée, sur les photos, une texture caractéristique facilement reconnaissable par les algorithmes de traitement d'images. La dynamique dans le temps et dans l'espace du glacier rocheux du Laurichard (figure 2.1; parc national des Écrins, Hautes Alpes) est relativement bien connue, grâce à un suivi en cours depuis 1984 (Bodin et al., 2009) ainsi que par des levés par LiDAR terrestre et par photogrammétrie (Bodin et al., 2018). La vitesse moyenne annuelle observée là où le glacier présente la plus grande pente est de l'ordre du mètre par an.

\subsection{Segmentation du champ de déplacement}

Le glacier rocheux du Laurichard (600 $\mathrm{m}$ de long pour 80 à $200 \mathrm{~m}$ de large) est instrumenté avec un appareil photo reflex Nikon D800 (capteur plein format, $36 \mathrm{Mp}$, 7360 x 4912 pixels) avec une focale de $85 \mathrm{~mm}$, situé à 300 mètres du glacier et enregistrant 8 images par jour. Ici, nous exploitons une série de 469 images prises entre le 7 juillet et le 22 septembre 2016, les images comportant trop d'artefacts (brouillard, neige, ombres trop importantes) ayant été retirées de la série. Ensuite une image par jour est sélectionnée manuellement en choisissant la plus favorable à l'algorithme calculant le déplacement. II s'agit le plus souvent d'une image sans soleil direct ne comportant pas d'ombres projetées, qui au cours de la journée peuvent dramatiquement changer l'aspect du glacier dans les images.

Les couples d'images pour le calcul du déplacement selon la méthode présentée dans la section 2.1 sont sélectionnés de manière à ce que le déplacement du glacier soit équivalent ou supérieur aux déplacements causés par le mouvement de la caméra. Dans le cas de Laurichard, une analyse empirique des déplacements dans les images a montré que le pas de temps minimum pour que le déplacement du glacier soit supérieur au déplacement provenant du mouvement de la caméra est d'une quarantaine de jours. Ainsi nous avons formé 612 couples d'images différents respectant un intervalle de temps supérieur à 40 jours.

Le coefficient de cohérence du champ de vecteurs déplacements est ensuite calculé selon l'équation 2 , le résultat obtenu est ensuite binarisé de façon à conserver uniquement les pixels où le coefficient VVC supérieur à un seuil, seuil déterminé par une méthode de Huang. Enfin des opérations morphologiques de type fermeture et ouverture sont appliquées afin de réduire le bruit dans le masque formé après l'étape de binarisation. Nous obtenons ainsi un masque indiquant les zones en mouvement, correspondant bien à l'expertise géomorphologique proposée par (Bodin et al., 2009). Les résultats de ces différentes étapes sont présentés dans la figure 2. La section suivante présente l'influence des paramètres que sont la taille de fenêtre pour le calcul du coefficient VVC discret et la valeur du seuil pour discriminer les zones en mouvements des zones fixes.

\subsection{Influence de la taille de fenêtre et de la valeur du seuil}

Comme indiqué dans la section 3.1, le glacier rocheux du Laurichard est étudié depuis plus de 30 ans, il nous est donc possible de fournir une vérité terrain (déterminée manuellement) des zones en mouvements dans les images prises par l'appareil photo. La vérité terrain est présentée dans la figure 2.4. Grâce à cette vérité terrain il est donc possible de tester notre algorithme avec différents paramètres de taille de fenêtre $w$ pour le calcul discret de VVC et du seuil sur le coefficient VVC pour réaliser la segmentation et ainsi déterminer leur influence sur la qualité du résultat. Les courbes de sensibilité/spécificité, ou courbes «Receiver Operating Characteristic » (ROC) sont l'outil idéal dans le cas d'un classificateur binaire dépendant d'un seuil, afin de déterminer l'influence du seuil et des paramètres du classificateur. Cette courbe représente le taux de vrais positifs en fonction du taux de faux positifs, chaque point de la courbe étant obtenu pour un seuil donné. II est ensuite possible de comparer deux classifieurs ou un même classificateur avec différents paramètres en s'intéressant à l'aire sous la courbe : plus l'aire sous la courbe est importante plus le classificateur est performant. Dans le cas de Laurichard nous avons réalisé des courbes ROC en faisant varier la taille de fenêtre $w$ pour le calcul du VVC de 10 à 100 pixels. Les courbes sont présentées dans 


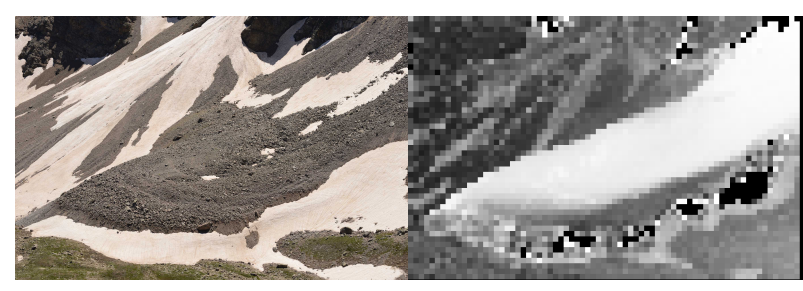

(1)

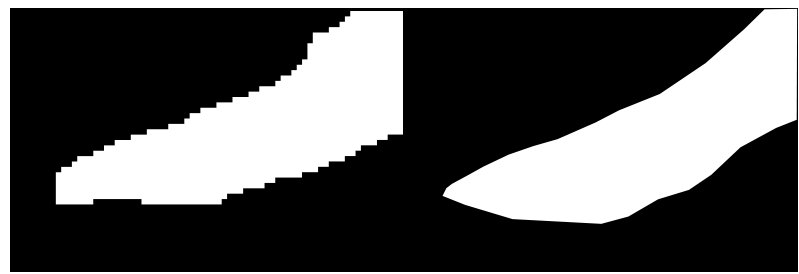

(3)

(4)

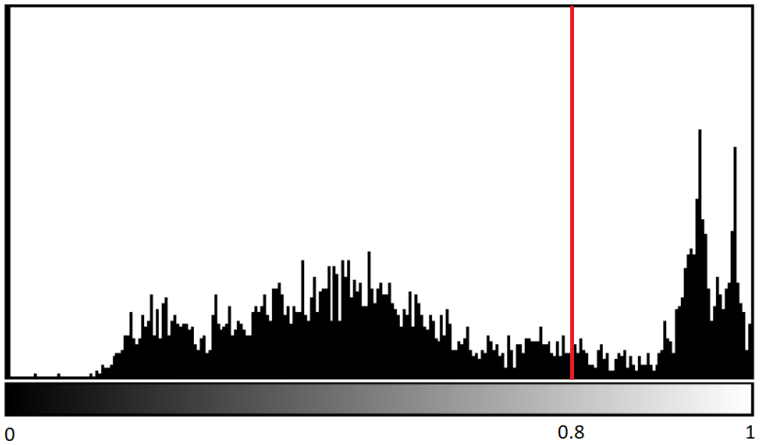

(5)

FIGURE 2: Segmentation automatique des zones en mouvements à partir du coefficient VVC sur une série d'images : (1) une image de la série; (2) le coefficient VVC calculé selon l'équation 2 sur une série de 469 images et 612 couples; (3) VVC après seuillage, binarisation et opérations morphologiques; (4) le masque correspondant à la vérité terrain réalisé manuellement. (5) Histogramme du coefficient VVC de la figure 2.2. Le seuil, déterminé par la méthode de Huang, est présenté en rouge. Ce seuil sépare les pixels fixes des pixels en mouvements.

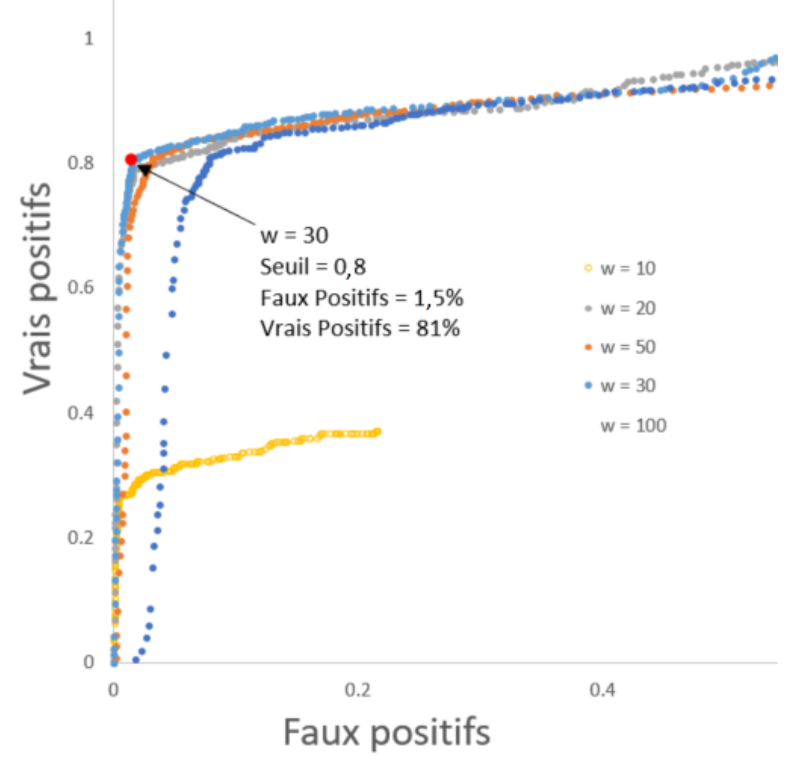

FIGURE 3: Courbes ROC de la segmentation de zones en mouvements à l'aide du coefficient VVC pour différentes tailles de fenêtres sur la série d'images du Laurichard. Le point rouge correspond à la configuration sélectionnée pour obtenir le masque des zones en mouvements utilisé pour estimer le déplacement réel du glacier.

la figure 3. Pour des tailles de fenêtres trop faibles (autour de 10 pixels) les opérations morphologiques appliquées ne permettent pas d'obtenir un taux de vrais positifs satisfaisant (taux inférieur à 0,4 ). Pour des tailles trop importantes (100 pixels) le taux de faux positifs est supérieur pour un taux de vrais positifs identiques à des tailles plus faibles (20, 30 pixels), cela indique que la segmentation est moins performante. Cela peut s'expliquer par le moyennage spatial trop important lors du calcul de VVC : les zones situées à la frontière entre les zones en déplacements et les zones fixes auront tendance à avoir un VVC moins important. Ces courbes montrent qu'une taille de fenêtre autour de 30 pixels semble adaptée dans le cas des images du Laurichard. Cela représente en moyenne une densité de 12 vecteurs déplacements par fenêtre pour chaque calcul de déplacement entre deux couples.

\subsection{Calcul du déplacement réel}

Pour corriger les déplacements de la caméra, une homographie est le plus souvent utilisée (Gance et al., 2014; Roncella et al., 2014; Hadhri et al., 2017) . En effet l'homographie est le modèle mathématique adapté pour modéliser une transformation projective (Hartley et Zisserman, 2004). La caméra étant fixe, on peut supposer que le déplacement observé des images provient principalement d'une rotation de la caméra. Ainsi dans le cas où une caméra est en rotation autour de son centre optique, l'ensemble des points des pixels des images avant et après rotation sont liés par une homographie, et ce quelque que soit la géométrie de la scène (Hartley et Zisserman, 2004). Une fois le masque obtenu, on peut 
donc utiliser les déplacements des zones fixes afin d'estimer une homographie (matrice $H 3 \times 3$ ) de telle sorte que, pour chaque pixel de correspondance de coordonnées $(x, y)$, subissant un déplacement $\left(\Delta_{u_{c}}, \Delta_{v_{c}}\right)$ du fait du mouvement de la caméra entre les images prises à $t_{0}$ et $t_{n}$, on obtienne :

$$
H\left[\begin{array}{l}
x \\
y \\
1
\end{array}\right]_{t_{0}}=\left[\begin{array}{c}
x+\Delta_{u_{c}} \\
y+\Delta_{v_{c}} \\
1
\end{array}\right]_{t_{n}} .
$$

Le champ de vecteurs déplacements étant bruité la méthode «RANdom SAmple Consensus » (RANSAC) (Fischler et Bolles, 1987) est utilisée afin d'écarter les déplacements aberrants. Le modèle utilisé pour le calcul du RANSAC est basé sur une estimation à l'aide de quatre points de correspondance, utilisant l'erreur de reprojection suivante, pour déterminer les déplacements aberrants :

$$
\begin{aligned}
e= & \sum_{i=0}^{N}\left(x_{i}+\Delta_{u_{c} i}-\frac{h_{11} x_{i}+h_{12} y_{i}+h_{13}}{h_{31} x_{i}+h_{32} y_{i}+h_{33}}\right)^{2} \\
& +\left(y_{i}+\Delta_{v_{c} i}-\frac{h_{21} x_{i}+h_{22} y_{i}+h_{23}}{h_{31} x_{i}+h_{32} y_{i}+h_{33}}\right)^{2}, \\
& h_{i j}: \text { coefficients de la matrice } H . \\
& N \text { : nombre de points de correspondance. }
\end{aligned}
$$

Une fois les déplacements aberrants détectés à l'aide du RANSAC l'ensemble des déplacements corrects sont utilisés pour estimer l'homographie finale par moindres carrés. Une fois l'homographie estimée elle est appliquée à l'ensemble des coordonnées des pixels de correspondance de l'image prise au temps $t_{0}$ afin de compenser le déplacement capteur $\left(\Delta_{u_{c}}, \Delta_{v_{c}}\right)$ et d'obtenir uniquement le déplacement du glacier $\left(\Delta_{u}, \Delta_{v}\right)$ :

$$
\left[\begin{array}{c}
\Delta_{u} \\
\Delta_{v} \\
1
\end{array}\right]=\left[\begin{array}{c}
x+\Delta_{u_{c}}+\Delta_{u} \\
y+\Delta_{v_{c}}+\Delta_{v} \\
1
\end{array}\right]_{t_{n}}-H\left[\begin{array}{l}
x \\
y \\
1
\end{array}\right]_{t_{0}}+\left[\begin{array}{l}
0 \\
0 \\
1
\end{array}\right]
$$

La figure 4 présente le résultat d'un calcul de champ de déplacement brut et ce même champ de déplacement après estimation de l'homographie et correction du champ de déplacement à l'aide de l'homographie. On remarque que les zones fixes ont un déplacement moins important après correction.

\section{5. Étude du déplacement du capteur}

Afin de mettre en évidence l'importance de l'étape de correction du déplacement, il est possible d'étudier le déplacement à l'intérieur de petites fenêtres situées sur des zones fixes et des zones mobiles. Pour des fenêtres suffisamment petites pour s'affranchir de l'effet de perspective dû à l'acquisition de l'image par une caméra, on s'attend à ce que la norme du déplacement brut moyen sur une fenêtre située sur une partie fixe soit non nulle à l'intérieur de la série et que son orientation varie au cours du temps. À l'inverse sur une série de déplacements recalés, la norme du vecteur moyen de cette même fenêtre doit être nulle, et ce pour l'intégralité de la série. Concernant une fenêtre sur le glacier, on s'attend à ce que la norme et la direction varient dans une série non recalée et soient peu significatives par rapport au déplacement des zones fixes pour une série non recalée, alors que dans le cas d'une série recalée, le déplacement du glacier doit être significatif au vu du déplacement des zones fixes.

Afin de mettre cela en évidence, nous avons sélectionné quatre zones de l'image pour lesquelles nous avons analysé le déplacement moyen au cours de la série d'images. Les zones sont représentées par les rectangles de couleurs de la figure 4, deux étant situées sur des zones fixes (vert en haut et rose en bas) et deux sur le glacier qui est mobile (orange en haut et rouge en bas). Un zoom sur le déplacement de ces zones pour différentes images de la série est présent dans la figure 5 . Nous avons ensuite analysé la variation de la norme du vecteur moyen de la fenêtre pour chaque fenêtre, dans différentes stratégies de correction du déplacement :

- sans correction,

- correction en estimant une homographie sur l'ensemble du champ de vecteurs,

- correction en estimant une homographie sur le champ de vecteurs masqués à l'aide du masque des zones mobiles issu du calcul du coefficient VVC,

- correction en estimant une homographie sur le champ de vecteurs masqués à l'aide du masque des zones mobiles issu de la vérité terrain.

Les résultats sont présentés dans le tableau 1. Dans le cas non recalé le déplacement moyen des zones fixes est de 4 pixels avec un écart type autour de 2 pixels, alors que le déplacement moyen du glacier et autour de 7 pixels, cela met bien en évidence l'importance de l'étape de correction du déplacement. Concernant la correction à l'aide d'une homographie estimée sur l'ensemble du champ de vecteurs, on observe une diminution du déplacement moyen de la zone fixe basse (de 4,2 à 1,4 pixels) mais une augmentation pour la zone fixe haute (de 4,0 à 9,1 pixels). II ne semble donc pas possible d'utiliser l'intégralité du champ de vecteurs pour estimer une homographie. En effet même si le RANSAC utilisé pour estimer l'homographie peut en théorie gérer un taux important de valeurs aberrantes, le déplacement du glacier, de par sa cohérence spatiale, ne peut pas être assimilé à des valeurs aberrantes par rapport au déplacement des zones fixes lors de l'estimation d'une homographie. Enfin dans le cas où les zones mobiles sont masquées à l'aide de la vérité terrain ou du coefficient VVC, on observe des résultats similaires, où le déplacement moyen des zones fixes est autour du pixel. Ces résultats valident donc l'utilisation d'une homographie estimée sur les déplacements des zones fixes pour corriger le déplacement de la caméra et recaler le champ de vecteurs. De plus l'utilisation d'un masque issu du calcul du coefficient VVC donne un résultat similaire sur les champs de vecteurs recalés à l'utilisation d'un masque issu de la vérité terrain. 


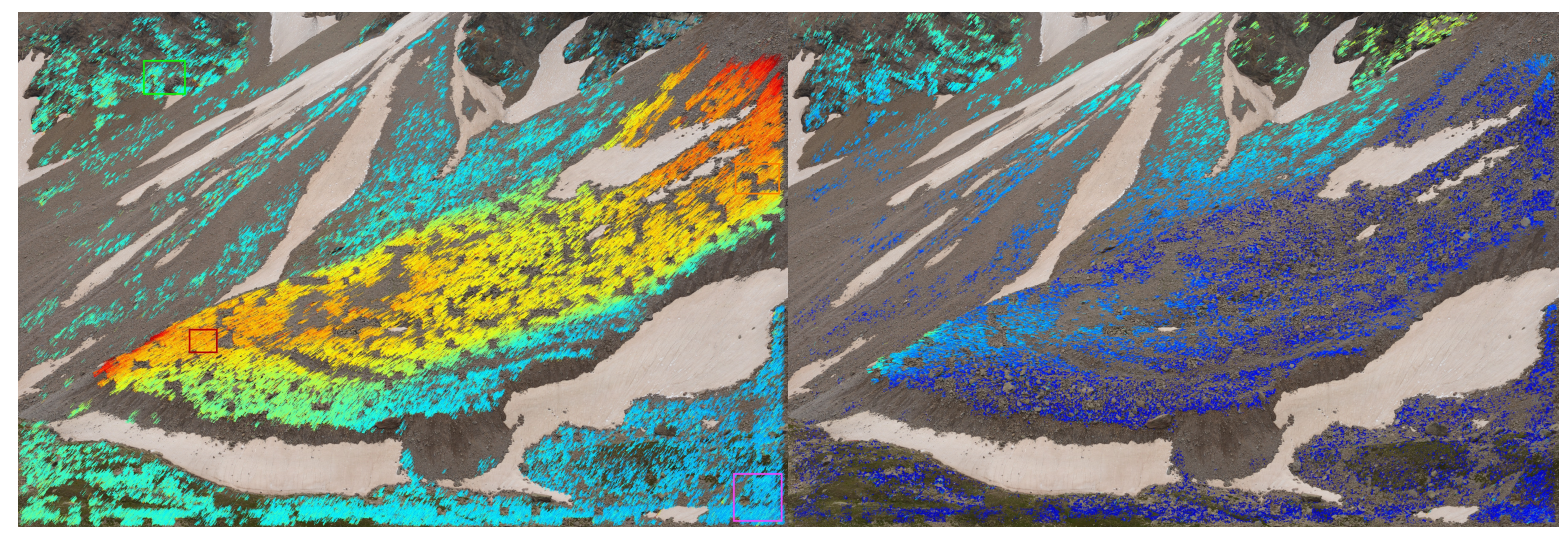

(1)

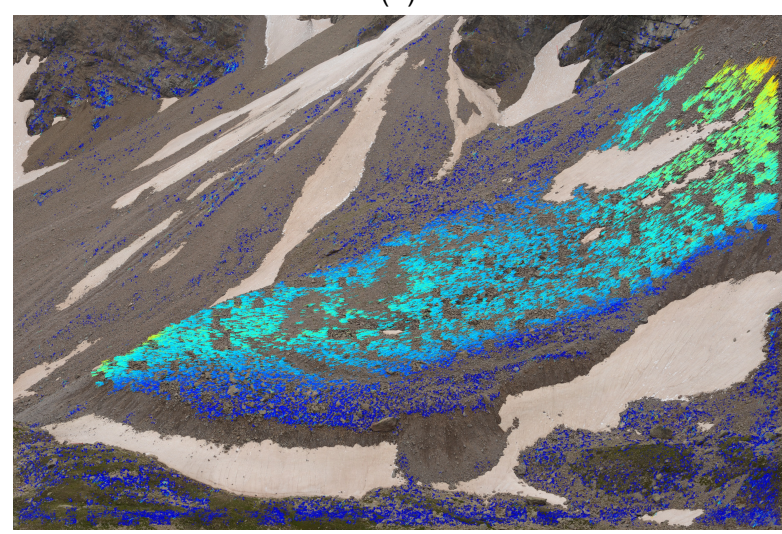

(3)
(2)

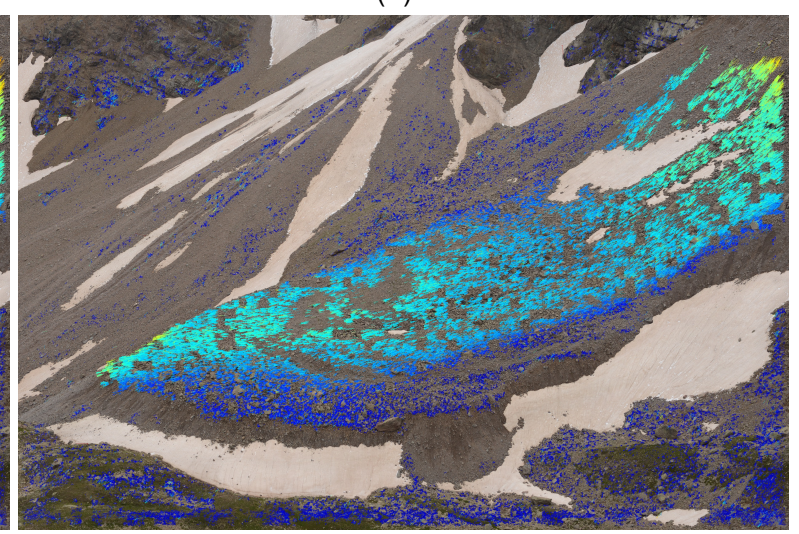

(4)

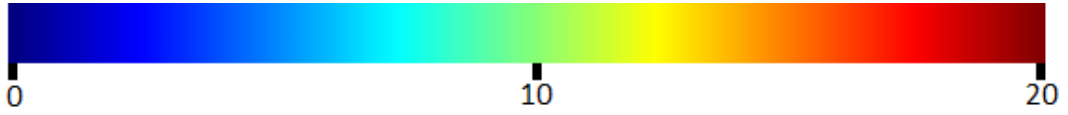

FIGURE 4: Champ de vecteurs déplacements (pixels) selon différentes stratégies de correction : (1) sans correction, (2) correction avec estimation de l'homographie sur l'ensemble du champ de vecteurs, (3) correction avec estimation de l'homographie sur le champ de vecteur masqué par le masque issu du calcul de VVC, (4) masque issu de la vérité terrain La taille de flèches, indiquant la norme du déplacement, a été multipliée par 5 pour une meilleure visualisation. 


\begin{tabular}{|c|c|c|c|c|c|}
\hline \multicolumn{2}{|c|}{} & \multicolumn{2}{|c|}{ Zone Fixe } & \multicolumn{2}{c|}{ Zone Mobile } \\
\cline { 3 - 6 } \multicolumn{2}{|c|}{} & Zone Basse & Zone Haute & Zone Basse & Zone Haute \\
\hline \multirow{2}{*}{ Brut } & Moyenne & 4,2 & 4,0 & 7,4 & 7,6 \\
\cline { 2 - 6 } & Ecart type & 1,8 & 2,1 & 2,2 & 2,3 \\
\hline \multirow{2}{*}{ Sans Masque } & Moyenne & 1,4 & 9,1 & 2,8 & 1,8 \\
\cline { 2 - 6 } & Ecart type & 1,3 & 5,5 & 2,0 & 1,8 \\
\hline \multirow{2}{*}{ Masque VVC } & Moyenne & 0,91 & 0,98 & 6,4 & 6,9 \\
\cline { 2 - 6 } & Ecart type & 0,76 & 1,1 & 1,5 & 1,7 \\
\hline \multirow{2}{*}{$\begin{array}{c}\text { Masque } \\
\text { vérité terrain }\end{array}$} & Moyenne & 0,90 & 0,98 & 6,4 & 6,8 \\
\cline { 2 - 6 } & Ecart type & 0,76 & 1,1 & 1,5 & 1,8 \\
\hline
\end{tabular}

TABLE 1: Analyse de l'influence de la correction des déplacements sur une série d'images sur la norme du déplacement moyen de fenêtres centrées sur des zones fixes et mobiles dans les différents cas : champ brut non recalé, champ recalé avec une homographie estimée sur l'ensemble du champ de vecteur, champ recalé à l'aide d'une homographie estimée sur le champ de vecteur masqué par le masque des zones mobiles issu du calcul de VVC, champ recalé de la même manière, mais en utilisant un masque issu de la vérité terrain.

\section{Conclusion}

Nous avons présenté une méthode permettant, à partir d'une série d'images non recalées, d'extraire automatiquement un masque indiquant les zones fixes des zones en mouvement. Une fois les couples d'images sélectionnés, les déplacements sont calculés sur l'ensemble de l'image pour chaque couple avec une méthode basée sur les détecteurs ORB. Une fois les champs de vecteurs calculés, leur cohérence temporelle est analysée à l'aide du coefficient VVC. Les zones de l'image ayant une forte cohérence correspondent à des zones en mouvements. Un masque indiquant les zones mobiles peut ainsi être calculé à l'aide du coefficient VVC. Afin de valider la méthode, nous avons analysé une série de 612 paires d'images du glacier rocheux du Laurichard entre juillet et septembre 2016. Une fois le masque extrait par la méthode présentée, nous avons compensé le déplacement de la caméra au cours de la série d'images en estimant une homographie à partir des déplacements des zones fixes. En analysant la chronique de déplacements moyens sur des fenêtres centrées sur des zones fixes et mobiles, nous avons montré que le déplacement de la caméra au cours du temps est une réalité et que l'étape de correction du déplacement est essentielle pour effectuer un suivi d'un versant de montagne. Par ailleurs, le masque des zones fixes et zones mobiles calculé par notre méthode est suffisant pour ensuite réaliser cette étape de correction. Nos futurs travaux porteront sur l'évaluation de la précision du calcul du déplacement en se confrontant à une vérité terrain. Afin de pouvoir comparer notre méthode à une vérité terrain, nous devrons convertir le déplacement pixellique en un déplacement métrique, en utilisant un modèle numérique de terrain ou en faisant appel à un second appareil photo.

\section{Références}

Bay, H., Tuytelaars, T., Gool, V., 2006. Surf : Speeded up robust features. Dans : European Conference on Computer Vision. Graz, Austria. pp. 404-417.

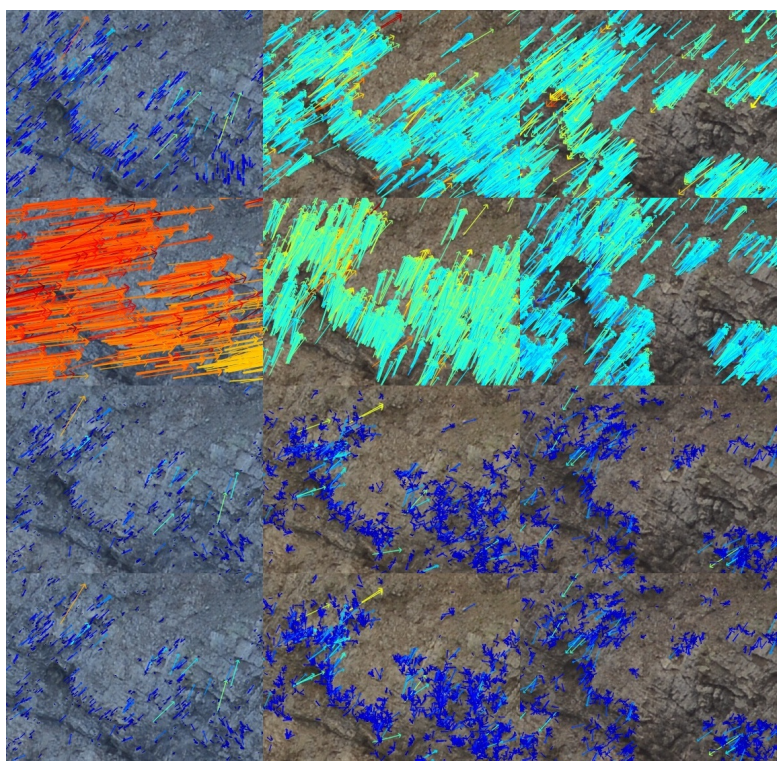

(1)

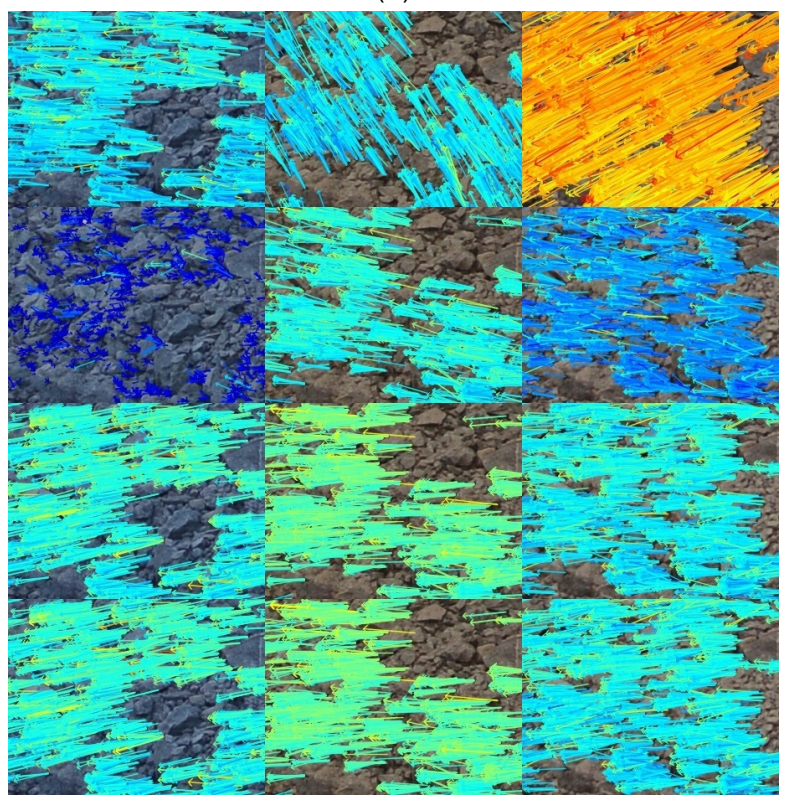

(2)

FIGURE 5: Détail du déplacement pour différents couples de la série d'images, selon différentes stratégies de correction du déplacement : sans correction (première ligne). Avec correction et estimation de l'homographie : sur l'ensemble du champ de vecteurs (seconde ligne), sur le champ de vecteur masqué par le masque issu du calcul de VVC (troisième ligne), masque issu de la vérité terrain (dernière ligne). (1) zone fixe en vert dans la figure 4 , (2) zone en mouvement en rouge dans la figure 4. 
Bodin, X., Thibert, E., Fabre, D., Ribolini, A., Schoeneich, P., Francou, B., Reynaud, L., Fort, M., 2009. Two decades of responses (1986-2006) to climate by the Laurichard rock glacier, French Alps. Permafrost and Periglacial Processes 20 (4), 331-344.

Bodin, X., Thibert, E., Sanchez, O., Rabatel, A., Jaillet, S., 2018. Multi-Annual Kinematics of an Active Rock Glacier Quantified from Very High-Resolution DEMs : An Application-Case in the French Alps. Remote Sensing 10 (4), 547.

Dehecq, A., Gourmelen, N., Trouve, E., 2015. Deriving largescale glacier velocities from a complete satellite archive : Application to the Pamir-Karakoram-Himalaya. Remote Sensing of Environment 162, 55-66.

Fischler, M. A., Bolles, R. C., 1987. Random sample consensus : a paradigm for model fitting with applications to image analysis and automated cartography. Dans : Readings in computer vision. pp. 726-740.

Gance, J., Malet, J.-P., Dewez, T., Travelletti, J., 2014. Target Detection and Tracking of moving objects for characterizing landslide displacements from time-lapse terrestrial optical images. Engineering Geology 172, 26-40.

Hadhri, H., Vernier, F., ATTO, A. M., Trouvé, E., 2017. Traitement automatique de time lapse : application à la surveillance de glaciers alpins. Dans : ORASIS 2017, journées francophones des jeunes chercheurs en vision par ordinateur. Collevillesur-Mer, France.

Haeberli, W., Hallet, B., Arenson, L., Elconin, R., Humlum, O., Kääb, A., Kaufmann, V., Ladanyi, B., Matsuoka, N., Springman, S., Mühll, D. V., 2006. Permafrost creep and rock glacier dynamics. Permafrost and Periglacial Processes 17 (3), 189-214.

Harris, C., Stephens, M., 1988. A combined corner and edge detector. Dans : In Proc. of Fourth Alvey Vision Conference. pp. 147-151.

Hartley, R., Zisserman, A., 2004. Multiple View Geometry in Computer Vision. Cambridge University Press.

Huang, L.-K., Wang, M.-J. J., 1995. Image thresholding by minimizing the measures of fuzziness. Pattern Recognition 28 (1), 41-51.

Lowe, D. G., 1999. Object recognition from local scale-invariant features. Dans : Proceedings of ICCV. Kerkyra, Greece. Vol. 2. leee, pp. 1150-1157.

Lowe, D. G., 2004. Distinctive image features from scaleinvariant keypoints. International Journal of Computer Vision 60 (2), 91-110.

Neyer, F., 2016. Monitoring Rock Glaciers by Combining Photogrammetric and GNSS-Based Methods. Thèse de doctorat, ETH Zurich, Suisse.

Pham, H. T., 2015. Analysis of optical stereo time lapse and radar satellite images : application to the measurement of glacier displacement. Thèse de doctorat, Université Grenoble Alpes, France.

Roncella, R., Forlani, G., Fornari, M., Diotri, F., 2014. Landslide monitoring by fixed-base terrestrial stereo-photogrammetry. ISPRS Annals of Photogrammetry, Remote Sensing and Spatial Information Sciences II-5, 297-304.

Rublee, E., Rabaud, V., Konolige, K., Bradski, G., 2011. ORB : An efficient alternative to SIFT or SURF. Dans : Proceedings of ICCV. Washington, DC, USA, pp. 2564-2571. 